\title{
Solar radius variations: new look on the wavelength dependence
}

\author{
Jean-Pierre Rozelot ${ }^{1}$, Alexander Kosovichev ${ }^{2}$ and Ali Kilcik ${ }^{3}$ \\ ${ }^{1}$ Université de la Côte d'Azur, CNRS-OCA, Nice cedex 4, France \\ email: jp.rozelot@orange.fr \\ ${ }^{2}$ New Jersey Institute of Technology, Newark, NJ 07103, USA \\ email: sasha@bbso.njit.edu \\ ${ }^{3}$ Department of Space Science and Technologies, Akdeniz University, 07058 Antalya, Turkey \\ email: alikilcik@akdeniz.edu.tr
}

\begin{abstract}
The possibility that the Sun's radius is changing, even at a faint level, has been discussed over a long time. As the solar radius is certainly one of the most important basic pieces of astrophysical information, it is crucial to determine the physical mechanisms that may cause shrinking or expansion of the solar envelope. The wavelength dependence has been poorly inspected up to now. Here we examine recent solar radius determinations from space observations, mainly from Mercury and Venus transits, made by different teams in 2006, 2012 and 2014. Seemingly, the results are not consistent: authors interpreted the discrepancies because of the different methods of analysis used in their work. However, looking at the wavelength dependence, adding other available observations, from X-EUV up to radio, a typical relationship between the radius and the wavelength can be found, reflecting the different heights at which the lines are formed. Possible explanations are discussed. Such results can be interesting for studying solar-stellar connections.
\end{abstract}

Keywords. astrometry, Sun: general, Sun: fundamental parameters, Sun: photosphere, Sun: radio radiation

\section{Measuring the solar diameter - a great deal of human efforts in the past}

The most obvious object visible in the sky is the Sun, and the earlier systematic observations were made by the Babylonian civilizations. However, these observations were concerning the motion of the Sun in the sky and not measurements of its size. One must wait for the Greeks astronomers to get a first assessment of the solar diameter. Aristarchus of Samos (circa 310-230 BC), by using a brilliant geometric procedure, was able to determine the solar diameter as the 720 th part of the zodiacal circle, i.e. $D_{\odot}=$ 1800 second of arc (') $\left(360^{\circ} / 720\right)$. A few years latter, Archimedes (circa 287-212 BC) wrote in the Sand-reckoner that the apparent diameter of the Sun appeared to lie between the 164 th and the 200 th part of the right angle, and so, $D_{\odot}$ must be estimated between $1620^{\prime \prime}$ and $1976^{\prime \prime}$ (respectively $27^{\prime} 00^{\prime \prime}$ and $32^{\prime} 56^{\prime \prime}$ ) (Shapiro (1975)) and (Lejeune (1947)). These values, albeit a bit erroneous are not too far from the most recent determinations, indicating by passing the great skillfulness of the Greek astronomers. A complete history of the solar diameter determinations can be found for example in Rozelot \& Damiani (1998). 


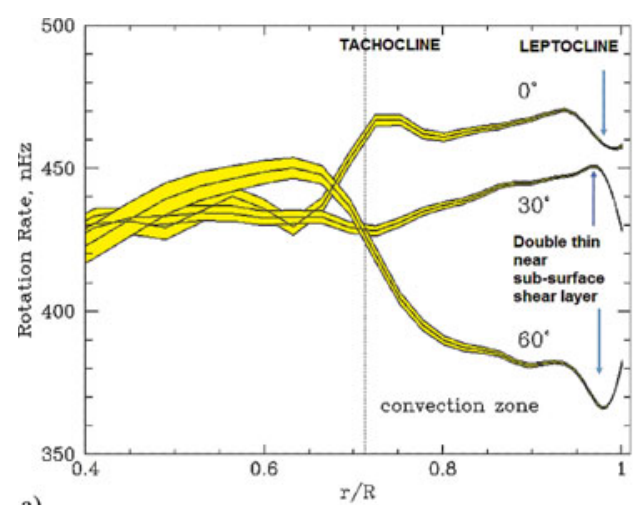

a)

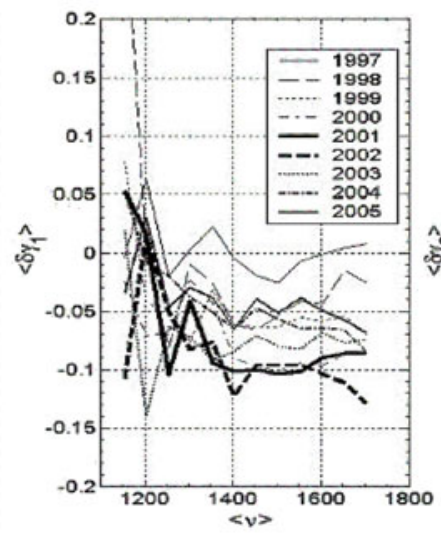

c) $\quad \gamma_{1}<0$ antiphase

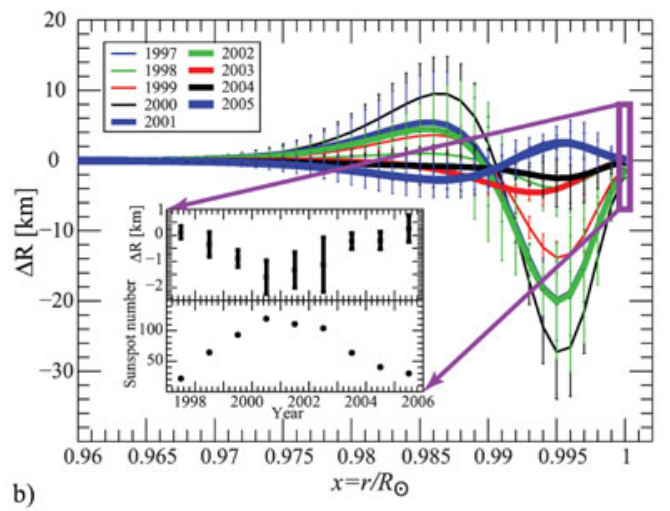

b)
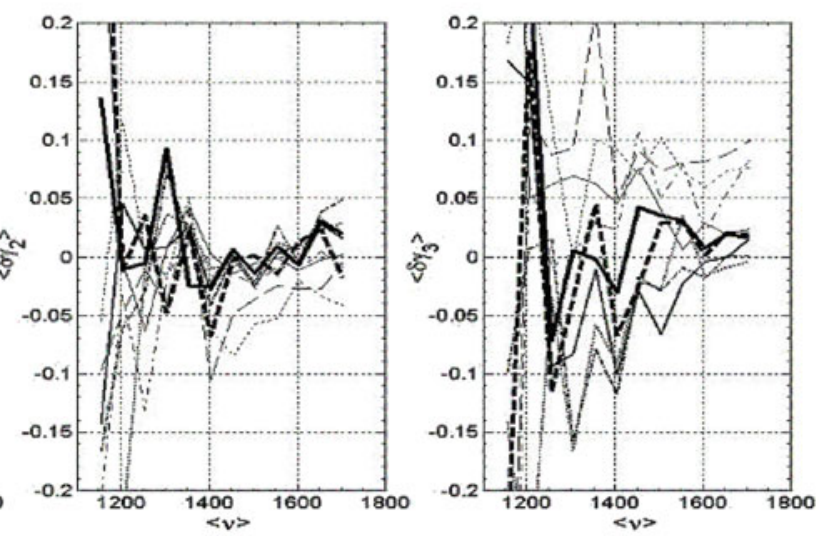

$\gamma_{2}$ positive trend

Figure 1. a) The rotation rate determined by helioseismology (Kosovichev et al. 1997) indicates a break near the surface (arrow). b) $f$-modes analysis show a non monotonic expansion of the solar radius with depth and a phase change with activity (Lefebvre et al. 2007). c) Three first asphericities parameters $\gamma$, i.e. even-a coefficients of $f$-modes. The layer around $0.995 R_{\odot}$ (called leptocline) is the seat of numerous physical changes. After Rozelot et al. (2006).

\section{A fundamental astrophysical quantity: the Solar Diameter}

Even if the absolute value of the solar diameter (or radius $R_{\odot}$ ) is not yet known accurately, the stellar radii are expressed in units of this quantity. Hence the need to get the most exact determination of the solar radius, or, referring to the conclusions of this study, the absolute necessity to quote the wavelength at which this determination has been made. The main concerns for such a determination can be listed as followed.

2.1. In solar physics, a change in the solar size is indicative of a change in the potential energy which could be driven by such means. To first order, a change in the solar radius carries luminosity changes, that are given by the Stephan's law, $L=\sigma T^{4}$, which gives in turn $\Delta L / L=4 \Delta T / T+2 \Delta R_{\odot} / R_{\odot}$, where $L$ is the solar irradiance, and $T$ is the solar effective temperature. Taking $L=1361 \mathrm{~W} / \mathrm{m}^{2}, T=5772 \mathrm{~K}$ (as recommended by the IAU) and $\Delta L=1.36 \mathrm{~W} / \mathrm{m}^{2}$ (i.e. $\Delta L / L \approx 0.01 \%$ as deduced from space observations (Scafetta \& Willson $(2014)$ ), it turns out that $\Delta R_{\odot}=7.3$ mas $(5.3 \mathrm{~km})$ if $\Delta T \approx 1.42 \mathrm{~K}$ over the solar cycle as found by Caccin \& Penza (2003). More refined computations lead to about the same conclusions, indicating that there is a faint cycle dependence. Such 
result is not surprising. Dziembowski, Goode \& Schou (2001) calculated the seismic radius shrinkage of about 2-3 km/year with rising activity. Goode \& Dziembowski (2003) using MDI (SOHO) high degree modes found a shrinking of the solar surface/convection zone (which seems to be cooler) with increasing activity, at a level consistent of the direct radius measurements based on $\mathrm{SOHO} / \mathrm{MDI}$ intensity data. Lastly, using a self-consistent approach taking into account an oblate Sun, Fazel et al. (2007) obtained an upper limit on the amplitude of cyclic solar radius variations (shrinking) between 3.87 and $5.83 \mathrm{~km}$, deduced from the gravitational energy variations.

2.2. Precise limb shape (curvature) changes both in latitude and time as an aspherical thermal structure. Such alterations play a role in the physics of the sub-surface layers. According to the $f$-mode frequencies measurements the temporal variations of the very near solar surface are stratified in a thin double layer, interfacing the deeper convective zone and the surface (Fig.1). This shear layer called "leptocline" (from the Greek "leptos": thin and "klino": hill) is the seat of many phenomena: an oscillation phase of the seismic radius, together with a non monotonic expansion of this radius with depth (Fig. 1b), a change in the turbulent pressure, likely an inversion in the radial gradient of the rotation velocity rate at about $50^{\circ}$ in latitude, opacities changes, superadiabicity, the cradle of hydrogen and helium ionization processes, and, probably, the seat of in-situ magnetic fields (Lefebvre et al. (2006)). Recent analysis of the high-degree oscillation modes revealed a sharp gradient of the sound speed in a narrow $30-\mathrm{Mm}$ deep layer just beneath the solar surface (Reiter et al. (2015)). The complex physics of this near surface shear zone (the leptocline) presumably plays an important role in the solar dynamo (Pipin \& Kosovichev (2011)). To this respect, new features of the SDO/HMI analysis is that the HMI data allow us to reconstruct the flows in this shallow subsurface layer, and match these to the directly observed surface flows. Such flows maps permit to investigate other important properties of the subsurface dynamics of the Sun, which previously were not accessible (Kosovichev (2016)). These results show that the latitudinal variations of the meridional circulation, which, presumably, affect the magnetic flux transport to the polar regions, occur in a relatively shallow subsurface layer (Fig. 2).

2.3. Temporal solar size variations, even faint, imply a dynamical gravitational moment, to first orders, $\mathrm{J}(2)$ and $\mathrm{J}(4)$. Precise knowledge of such quadrupole moments are required to develop high precision astrometry and in addition, may constraint gravitational theories both on a theoretical and experimental point of views. In such prospect, the Eddington-Robertson parameters, $\gamma$, and $\beta$ contributes to the relativistic precession of planets. Note that $\gamma$ encodes the amount of curvature of space-time per unit restmass, and the post-Newtonian parameter $\beta$ encodes the amount of non-linearity in the superposition law of gravitation. It is still difficult to disentangle $\mathrm{J}(2), \gamma$ and $\beta$. However, by accurately measuring the limb curvature over the latitudes, -that is to say the solar oblateness-, it is possible to get a good estimate of the solar quadrupole moment, to an accuracy of one part in 200 of its size of around $10^{-7}$. Recent analysis include the LenseThirring precession effect, which is not negligible, as in the case of Mercury for instance, it may have been canceled to a certain extent by the competing precession caused by a small inaccuracy in the quadrupole mass moment $J(2)$ of the Sun (Iorio (2011)).

\section{Data collections}

The importance of limb shape dependence on the wavelength was recognized decades ago, mainly through the pioneering works of Pierce \& Slaughter (1977) and Pierce 

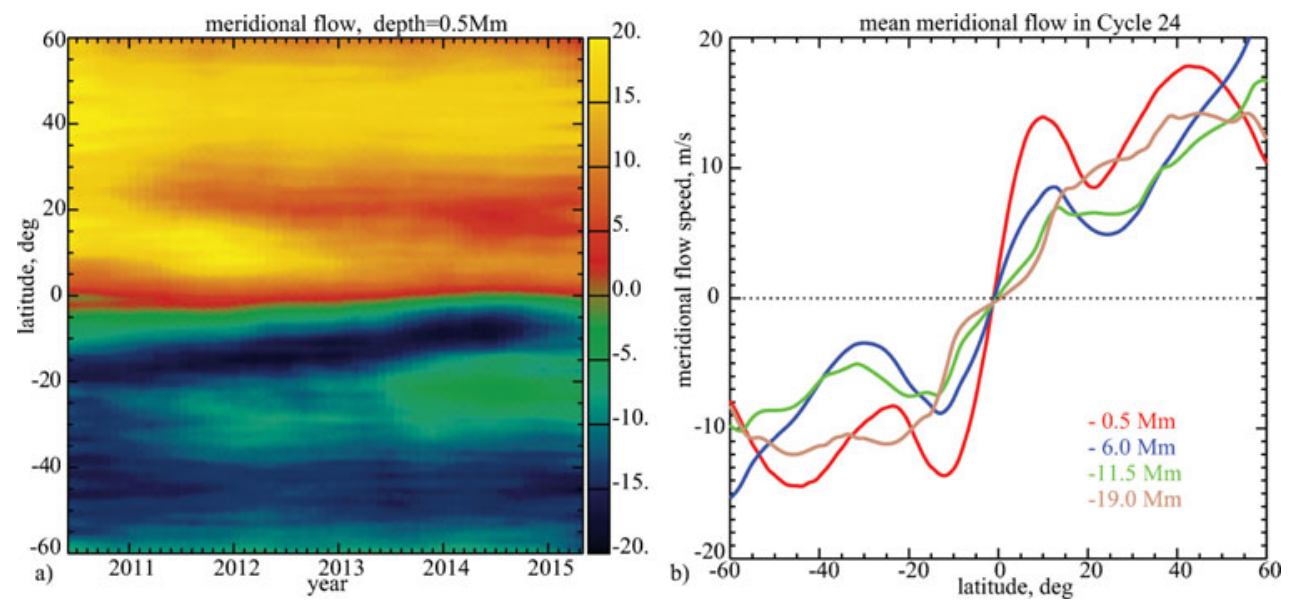

Figure 2. a) Evolution of the subsurface meridional flows obtained from the 5-years of the SDO/HMI observations during Solar Cycle 24. The red and yellow colors show the flow components towards the North pole, the green and blue colors show the South-ward flow. The color scale range is from -20 to $20 \mathrm{~m} / \mathrm{s}$. b) The mean meridional flow averaged for the whole period of observations at four different depths. Kosovichev \& Zhao (2016).

et al. (1977), then by Neckel \& Labs $(1987,1994)$ who analyzed the limb darkening from $303 \mathrm{~nm}$ up to $2400 \mathrm{~nm}$. They found that the limb-darkening function could be fitted by a fifth order polynomial with no significant variations during the solar cycle. Since then, few solar radius measurements with wavelength have been made.

\subsection{From space observations.}

In the UV part of the spectrum, data are coming from the Extreme Ultraviolet Imager (EIT) aboard the SOHO spacecraft (Delaboudinière et al. (1995)) and analyzed by Selhorst, Silva \& Costa (2004). In the near infra-red, the Michelson Doppler Imager (MDI) aboard the Solar and Heliospheric Observatory (SOHO) has been used to observe the Mercury transit in 2003 and 2006 and the Venus transit in June 2012. The HMI/SDO images (Helioseismic and Magnetic Imager instrument -HMI- aboard the Solar Dynamics Observatory) (Scherrer et al. (2012)) provided data during the 2012 Venus transit analyzed by two separate teams, in the near infra red, whilst the AIA (Atmospheric Imaging Assembly) (Lemen et al. (2012)) instruments aboard the SDO provided data in the near UV field, always during the 2012 Venus transit. Lastly, the Solar Disk Experiment (SDS) embarked on-board balloon flights enable us to enrich the data in the near IR (Sofia et al. (2013)).

\subsection{From ground-based observations.}

An investigation of the existing literature shows that the solar radius has been observed in specific wavelengths, by means of the so-called Picard-sol instrument installed at the Calern observatory, South of France (Meftah et al. (2014)), and by the help of the Heliometer installed at the Pic du Midi Observatory (South of France) (Rozelot et al. (2013)). Sigismondi et al. (2015) observed the Venus transit in 2004 in Athens by means of a $\mathrm{D} / \mathrm{f}=120 / 1000$ refractor and were able to measure the solar radius in $H_{\alpha}$.

The solar limb has been observed in X-rays by means of the RHESSI (The Reuven Ramaty High Energy Solar Spectroscopic Imager) satellite and results can be found in Hudson \& Battaglia (2014). They determine the limb heights of four estimates provided by different authors, above (or below) the referenced atmosphere at $\tau_{500}=1$ (the 
canonical value of Auwers (1891)). These values can, in turn, be transformed into radius, leading to a relationship given by $\mathrm{R}=0.00003 x^{2}-0.03583 x+969.39850$, where $x$ is the wavelength in $\mathrm{nm}$ (with a correlation coefficient of $\mathrm{r}=0.99$ ). Taking into account the RHESSI estimate, at $1 \mathrm{~nm}$, this expression leads to $\mathrm{R}=969^{\prime \prime} .36$ perfectly compatible with the $969^{\prime \prime} .01$ deduced from our quadratic fit as shown in Fig. 4. The RHESSI estimate deduced by (Hudson \& Battaglia (2014)) is less than this value, but is also misleading without the corresponding wavelength. Moreover, RHESSI was not really designed for the purpose of measuring the solar diameter. Obviously, the above-mentioned relationship is a bit crude and cannot be extrapolated in the IR as the solar atmospheric parameters are quite different.

In the radio band, several determinations of the solar radius have been made by radio telescopes at millimeter waves, including eclipse observations at centimeter and decimeter waves, and interferometric observations at meter waves (see, for instance, Table 5 in Benz (2009)). The solar radius in radio waves may be defined as the radius of the isophote $T_{\text {rad }}$ $=0.5 T_{c}$ at the limb, where $T_{r a d}$ is the brightness temperature and $T_{c}$ the brightness temperature at the center of the solar disk. Both the equatorial and the polar radii increases, starting around $10 \mathrm{~mm}$, which are due to the coronal contribution. Observations of the solar radius have been made from 0.7 to $35 \mathrm{~mm}$ (i.e. 404 to $8.5 \mathrm{GHz}$ ) by different authors (Kalaghan \& Telford (1970); Bachurin (1984); Selhorst et al. (2004); Giménez de Castro et al. (2007); Benz (2009)) and are plotted in the right side of Fig. 3 (composite of different data). As shown in this figure the solar radius continuously increases in the millimeter wavelength range due to the coronal contribution.

\section{Overview}

An overview of the currently known inventory of solar radius with wavelength is presented in Table 1.

A second-order polynomial fits the data, showing a strong wavelength dependence of the solar radius. However, a large wavelength domain from 667 to $742,060 \mathrm{~nm}$ is currently unexplored. In this range the polynomial fits suggests a minimum in the mid-IR region at about $6.6 \mu \mathrm{m}$. The same fitting curves deduced with the higher and lower error bars allow us to deduce an uncertainty of $1.9 \mu \mathrm{m}$. Albeit measurements were obtained at different periods of time, no significant radius temporal variations can be found, at least at the level of the uncertainty at which observations were made.

No model can reproduce the entire spectrum today. Attempts have been made in the visible part (Thuillier et al. 2011), as well as in the radio part (Selhorst et al. (2009)). Considering the first domain, among the five solar models described, i.e. SH09, VAL81, COSI and FCH09 (see the paper for their description), only the FCH09, performed with the Solar Modelling in 3D (SolMod3D) code, may mimic the range of the observations, as seen in Fig. 3 taking into account the concavity of the curves. The figure shows the inflection point position calculated versus wavelength, in quiet conditions with respect to $\tau_{500}=1$, reflecting the limb shape displacement. However, the theoretical models still do not fully explain the measured variations (somewhat larger, as the theoretical range between 100 and $1000 \mathrm{~nm}$ is about $100 \mathrm{~km}$, or 0.14 arcsec, less than the 2 arcsec as detected by observations). The FCH09 model is different compared to other models mainly due to the temperature minimum, significantly colder than that taken in other models, and by the increase of the $\mathrm{CH}$ continuum opacity below $420 \mathrm{~nm}$, which account only in SolMod3D.

As far as the radio domain is concerned, the global models are still not satisfactory, mainly due to (i) the brightness temperature predicted by the atmospheric model, overes- 
Table 1. Summary of the Solar Radius Observations at Different Wavelengths.

\begin{tabular}{|c|c|c|c|c|}
\hline $\mid \begin{array}{c}\text { Wavelength } \\
{[\mathbf{n m}]} \\
{[\mathbf{G H z}]}\end{array}$ & 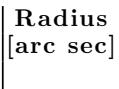 & $\left|\begin{array}{c}\text { Error } \\
{[\operatorname{arc} \text { sec }]}\end{array}\right|$ & Experiment & References \\
\hline 17.1 & 964.54 & 0.02 & EIT-SOHO & Gimenez de Castro et al. (2007) \\
\hline 30.4 & 967.56 & 0.039 & EIT-SOHO & Gimenez de Castro et al. (2007) \\
\hline 160.0 & 963.04 & 0.03 & AIA 2012 Venus transit & Emilio et al. (2015) \\
\hline 170.0 & 961.76 & 0.03 & AIA 2012 Venus transit & Emilio et al. (2015) \\
\hline 500.0 & 959.63 & 0 & Canonical value & Auwers (1891) \\
\hline 505.8 & 959.434 & 0.008 & Heliometer & Rozelot et al. (2003) \\
\hline 535.8 & 959.78 & 0.19 & $\begin{array}{c}\text { SODISM II } \\
\text { (ground-based) }\end{array}$ & Meftah et al. (2014) \\
\hline 607.1 & 959.86 & 0.18 & $\begin{array}{l}\text { SODISM II } \\
\text { (ground-based) }\end{array}$ & Meftah et al. (2014) \\
\hline 607.1 & 959.85 & 0.19 & $\begin{array}{l}\text { SODISM PICARD } \\
2012 \text { Venus transit }\end{array}$ & Hauchecorne et al. (2014) \\
\hline 615.0 & 959.76 & 0.12 & SDS & Sofia et al. (2013) \\
\hline 617.3 & 959.57 & 0.02 & HMI & \\
\hline & & & 2012 Venus transit & Emilio et al. (2015) \\
\hline 617.3 & 959.90 & 0.06 & $\begin{array}{l}\text { SODISM PICARD } \\
2012 \text { Venus transit }\end{array}$ & Emilio et al. (2015) \\
\hline $656.281\left(\mathrm{H}_{\alpha}\right)$ & 960.017 & 0.009 & 2004 Venus transit & Sigismondi et al. (2015) \\
\hline 676.78 & 960.12 & 0.09 & $\begin{array}{l}\text { MDI } 2003 \\
\text { and } 2006 \text { Mercury transits }\end{array}$ & Revisited value Kuhn et al. (2014) \\
\hline 7.5 & 1013.87 & 5.6 & & Benz (2009) \\
\hline 8.6 & 1021.43 & 5.0 & Radio Crimean Observatory & Bachurin (1983) \\
\hline 10.0 & 999.96 & 5.6 & & Benz (2009) \\
\hline 12.0 & 1003.77 & 5.0 & Radio Crimean Observatory & Bachurin (1983) \\
\hline 15.0 & 988.84 & 5.6 & & Benz (2009) \\
\hline 16.0 & 993.18 & 5.0 & Radio Crimean Observatory & Bachurin (1983) \\
\hline 17.0 & 976.50 & 1.5 & Nobeyama Radioheliograph & Selhorst (2011) mean value on the graph \\
\hline 22.0 & 985.00 & 6.0 & $\begin{array}{c}\text { Itapentiga Radio } \\
\text { Observatory (Brazil) }\end{array}$ & Selhorst (2010) \\
\hline 22.0 & 981.70 & 2.0 & Nobeyama Radioheliograph & Costa et al. (1985) cited by Costa (1999) \\
\hline 30.0 & 977.71 & 5.6 & & Benz (2009) \\
\hline 33.3 & 969.23 & 0.0 & $\begin{array}{c}\text { Interferometer, } \\
\text { University of Kent, Canterbury }\end{array}$ & Nicholson \& Parker (1973) \\
\hline 34.9 & 988.42 & 0.0 & & Kalaghan (1970), cited by Nicholson (1973) \\
\hline 37.5 & 976.32 & 5.6 & & Benz (2009) \\
\hline 43.0 & 981.00 & 6.0 & Nobeyama Radioheliograph & Selhorst (2010) \\
\hline 44.0 & 977.86 & 2.0 & Nobeyama Radioheliograph & Costa et al. (1985) cited by Costa (1999) \\
\hline 48.0 & 983.62 & 1.9 & Nobeyama Radioheliograph & Costa (1999) \\
\hline 50.0 & 976.32 & 5.6 & & Benz (2009) \\
\hline 75.0 & 980.49 & 5.6 & & Benz (2009) \\
\hline 150.0 & 974.93 & 5.6 & & Benz (2009) \\
\hline 212.0 & 972.00 & 3.0 & Nobeyama Radioheliograph & Selhorst (2010) \\
\hline 404.0 & 975.00 & 5.0 & Nobeyama Radioheliograph & Selhorst (2010) \\
\hline
\end{tabular}

Note: First column: in nm, first part of the Table; in GHz, second part of the Table. Benz (2009): different sources have been compiled, coming from Hachenberg, O., in: Landolt-Börnstein, New Series, Vol. VI/2a, Astronomy and Astrophysics (K. Schaifers, H. Voigt, eds.), Berlin, Heidelberg, New York: Springer-Verlag (1981) p. 106.

timated at high frequencies (212 and $405 \mathrm{GHz}$ ), needing to be changed in the photosphere and lower chromosphere; (ii) by the spicules temperatures and densities which should be revised to get a better fit with the observations.

Lastly, it could be argued that the solar data were collected at different period of time. Thus, in principle, it would be possible to disentangle with the solar activity. However on the one hand, there are not enough data available, specially in the UV and visible part of the spectrum, and in the other hand, as already seen, the temporal variations of the solar radius are not of enough amplitude to determine a significant different parabolic fit as those found in this first approach. 


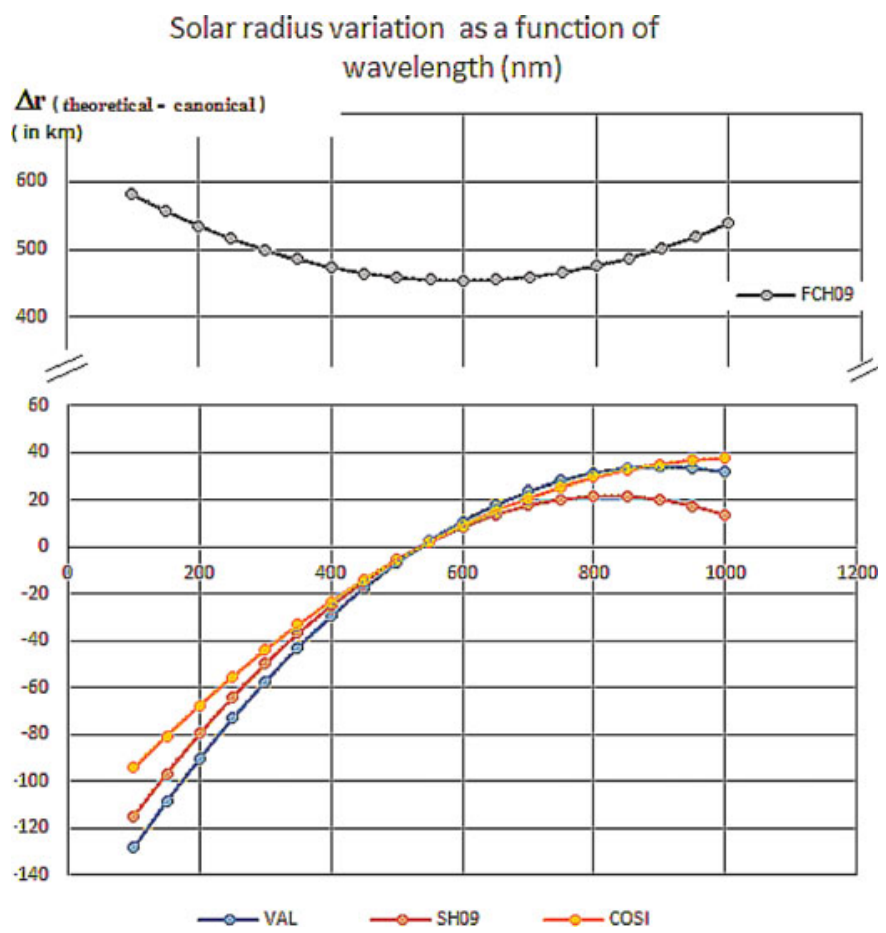

Figure 3. Solar radius variations (in $\mathrm{km}$ ) plotted versus the wavelength (in $\mathrm{nm}$ ), according to five models: VAL81 (based on Skylab observations of the quiet Sun, in the wavelength range 40-140 nm), SH09 (one-dimensional stellar atmospheric modeling and spectrum synthesis algorithm), COSI (radiative transfer code) and FCH09 (Solar Modeling in 3D). The ordinate scale $(\Delta r=r($ theoretical $)-r($ canonical $))$ is the difference between the computed radius and the canonical radius $(695508 \mathrm{~km})$. This difference corresponds also to the displacement of the inflection point of the limb shape intensity with respect to $\tau_{500}=1$. Only the FCH09 model may explain the variation of the solar radius with the wavelength in the visible domain (taking into account the positive second derivative). See Thuillier et al. (2011) for further details concerning the models.

\section{Conclusion}

The diameter of the Sun is certainly one of the most important astrophysical parameter. From time immemorial men have striven to get a measure of this diameter, which was a source of curiosity and study. Tackled by Greek astronomers from a geometric point of view, an estimate, although incorrect, has been first determined, not truly called into question for several centuries. One must wait up to the XVIIth century to get the first precise determinations made by the French school of astronomy lead by Mouton, Picard and La Hire. Since then, a number of techniques has been used mainly in England, Germany, Italy and US, all aiming at getting the most accurate value of the diameter of the Sun. However, even with instruments at the cutting edge of progress, no absolute value has been provided yet. The mean radius of $959^{\prime \prime} .63$ (i.e. $695997 \mathrm{~km}$ with the new IAU astronomical unit), as obtained by the German astronomer Auwers in 1891, has been adopted as a "canonical value", and is determined at the optical depth $=1$, i.e. at a wavelength of $500 \mathrm{~nm}$. But the precise location of the limb of the Sun depends upon the wavelength of observation. Hence, we pointed out here the need for accurate observations over the whole solar spectrum. Solar diameter determinations from space observation of Mercury and Venus transits have been made by different teams, in 2003, 2006, 2012 and 2014 (by means of SDO). Other measurements have been made from the Extreme 


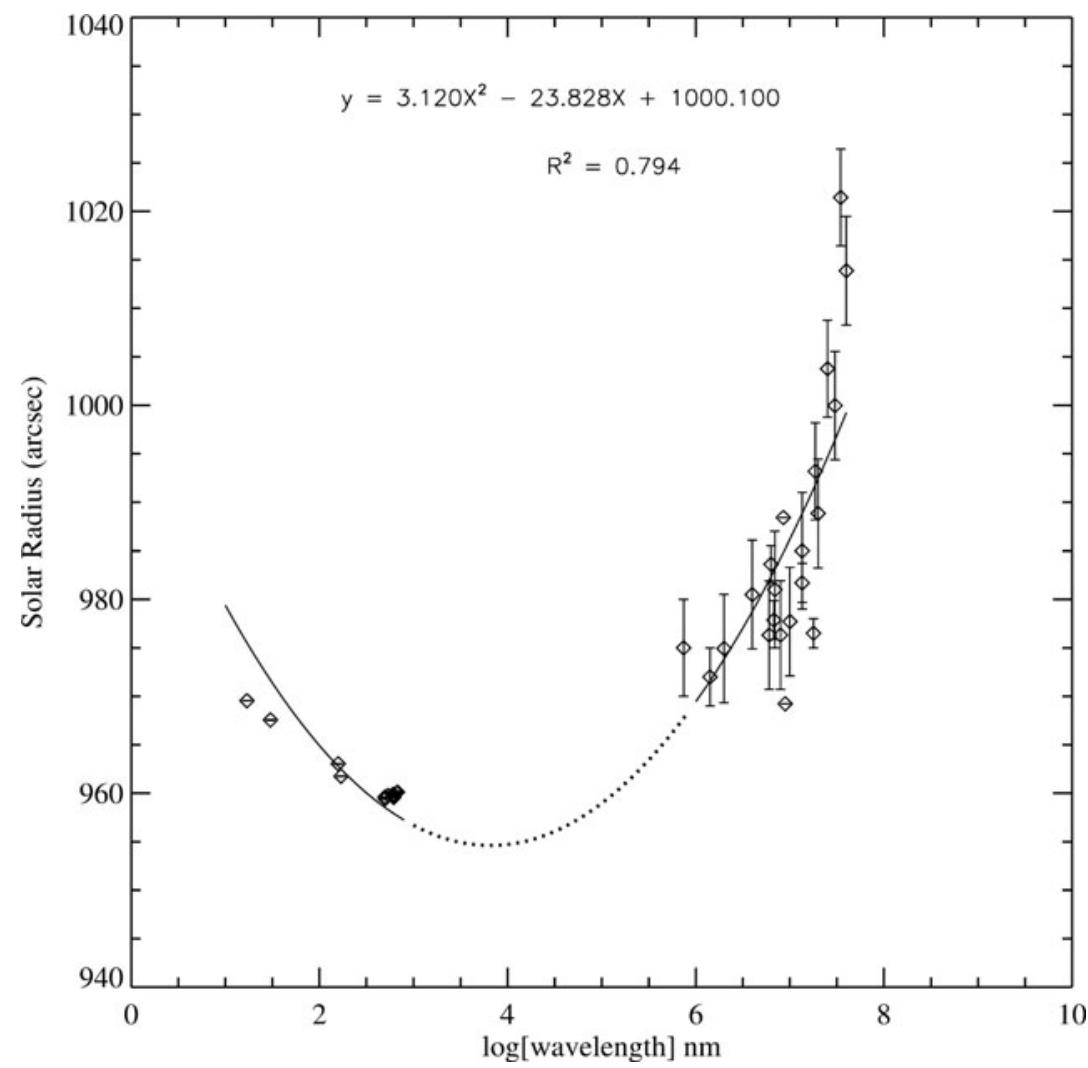

Figure 4. Solar radius variations from EUV to $H_{\alpha}$ (on the left side) to millimeter radio waves (on the right side) as a function of wavelength in the decimal logarithm scale. A second order polynomial correctly fits the data showing a strong wavelength dependence of the solar radius. The mid-domain (curve in dots) ranging from $677 \mathrm{~nm}$ to $742,060 \mathrm{~nm}(404 \mathrm{GHz})$ is presently still unexplored. A minimum is obtained for about $6.6 \mu \mathrm{m}$ with an estimated error o $\pm 1.9 \mu \mathrm{m}$. No unique model can currently explain such an important wavelength variation. See also Rozelot et al. (2015) for further descriptions.

Ultraviolet Imager (EIT) aboard the SOHO spacecraft, from the Solar Disk Sextant (SDS) embarked on balloon flights, from the heliometer at the Pic du Midi South France and from the Picard mission, both in space and on the ground. Adding radio data in the millimetric domain, a typical wavelength dependence has been found, reflecting the different heights at which the lines are formed. An unexpected minimum at around $(6.6 \pm$ 1.9) $\mu \mathrm{m}$ was obtained, located in a still unexplored domain. No unique theoretical model is available today to reproduce this strong wavelength dependence. Thus, our quest for precise measurements of the solar diameter will continue.

\section{Acknowledgements}

This work was supported in part by the NASA grant NNX14AB70G (A.Kosovichev), and by the Scientific Research Projects Coordination Unit of Akdeniz University (A. Kilcik). J.P. Rozelot would like to thank the International Space Science Institute (ISSI) in Bern $(\mathrm{CH})$ for its support of this study. 


\section{References}

Auwers, A. 1891, Astronomische Nachrichten, 128, 361

Bachurin, A. F. 1984, Bull.Crimean Astr. Obs., 65, 64

Benz, A. O. 2009, in Radio Emission of the Quiet Sun (Berlin: Springer)

Caccin, B. \& Penza, V. 2003, Mem. S.A.It. Vol. 74, 663

Delaboudinière, J.-P., Artzner, G. E., Brunaud, J., et al. 1995, Sol. Phys., 162, 291

Dziembowski, W. A., Goode, P. R., \& Schou, J. 2001, ApJ, 553, 897

Fazel, Z., Rozelot, J. P., Lefebvre, S., Ajabshirizadeh, A., \& Pireaux, S. 2008, New Astr., 13, 65

Goode, P. R. \& Dziembowski, W. A., 2003, Jour. of the Korean Astron. Soc., 36, S75-S81

Hudson, H. \& Battaglia, M. 2014, http://sprg.ssl.berkeley.edu/ tohban/wiki/index.php/The_Solar_X-ray_Limb_II

Iorio, L., Lichtenegger, H.I.M., Ruggiero \& M.L. Corda, C. 2011 Astrophys. Space Sci., 331, 351

Kosovichev, A. G., Schou, J., Scherrer, P. H., et al. 1997, Sol. Phys., 170, 43

Kosovichev, A. K. 2016, in Cartography of the Sun and the Stars, Lecture Notes in Physics, J.P. Rozelot \& C. Neiner, ed., Springer, 914, in press

Lefebvre, S., Kosovichev, A. G., Nghiem, P., Turck-Chièze, S., \& Rozelot, J. P. 2006, SOHO 18 Conference, Sheffield, U.K., August 7-11, 2006. "Beyond the Spherical Sun: a new era of helio-and asteroseismology". ESA-SP, 624, CDROM, 9.1

Lefebvre, S., Kosovichev, A. G., \& Rozelot, J. P. 2007, ApL Lett., 658, L135

Lejeune, A. 1947, Annales Société Scientifique de Bruxelles, Série 1 Vol. LXI, 27

Lemen, J. R., Title, A. M., Akin, D. J., et al. 2012, Sol. Phys., 275, 17

Meftah, M., Corbard, T., Irbah, A., et al. 2014, A $\&$ A, 569, A60

Nicholson, P. S. \& Parker, E. A. 1973, Obs, 93, 13

Pierce, A. K. \& Slaughter, C. D. 1977, Sol. Phys., 51, 25

Pipin, V. V. \& Kosovichev, A. G. 2011, ApJ, 727 L45

Reiter, J., Rhodes, E. J., Jr., Kosovichev, A. G., et al. 2015, ApJ, 803, 92

Rozelot, J. P., Lefebvre, S., \& Desnoux, V. 2003, Sol. Phys., 217, 39

Rozelot,J. P., Kosovichev, A. K. \& Lefebvre, S. 2006, Highlights of Astronomy, Vol. 14, K.A. von der Hucht ed.

Rozelot, J. P. \& Damiani, C. 2003, The Euro. Phys. Jour. H, 37, 709

Rozelot, J. P., Kosovichev, A., \& Kilcik, A. 2015, ApJ, 812, 91

Shapiro, A. E. 1975, Journal of Historical Astronomy, p. 75-80

Scafetta, N. \& Willson, R. 2014, Astr. Sp. Sc., 350, 421

Scherrer, P. H., Shou, J., Bush, R. I., et al. 2012, Sol. Phys., 275, 207

Selhorst, C. L., Silva, A. V. R., \& Costa, J. E. R. 2004, A \& A, 420, 1117

Sigismondi, C., Ayiomamitis, A., Wang, X., et al. 2015, http://de.arxiv.org/ ftp/arxiv/papers/1507/1507.03622.pdf

Sofia, S., Girard, T. M., Sofia, U. J., et al. 2013, MNRAS, 436, 2151

Thuillier, G., Claudel, J., Djafer, D., et al. 2011, Sol. Phys., 268, 125 